Original Research Paper

\title{
Efficient Production of Glycosylated and Non-Glycosylated Mycobacterium tuberculosis Antigen 85B Fused to PVX Coat Protein in Nicotiana benthamiana Plants
}

\author{
${ }^{1}$ Pablo A. González, ${ }^{1}$ Franco D. Puccio and ${ }^{1,2}$ Alicia M. Zelada \\ ${ }^{1}$ Laboratorio de Agrobiotecnología, DFBMC, FCEN, \\ Universidad de Buenos Aires, Ciudad Universitaria, 2do piso, 1428, Buenos Aires, Argentina \\ ${ }^{2}$ Instituto de Biodiversidad y Biología Experimental y Aplicada (IBBEA-UBA-CONICET), Buenos Aires, Argentina
}

Article history

Received: 04-08-2018

Revised: 30-09-2018

Accepted: 10-11-2018

Corresponding Author:

Alicia M. Zelada

Laboratorio de

Agrobiotecnología, DFBMC,

FCEN, Universidad de Buenos

Aires, Ciudad Universitaria,

2do piso, 1428, Buenos Aires,

Argentina

Email: azelada@fbmc.fcen.uba.ar

\begin{abstract}
The development of alternative subunit based-vaccines against tuberculosis is necessary due to variable efficiency and some security concerns of the BCG vaccine. The aim of this work was evaluate the production of the Mycobacterium tuberculosis Ag85B antigen fused to Potato Virus $X$ Coat Protein (PVX-CP) by transient expression in Nicotiana benthamiana for subunit-based tuberculosis vaccine formulation. A codonoptimized M. tuberculosis Ag85B gene was fused to PVX-CP and expressed both as a full length precursor and as a mature version lacking the leader peptide. Signal peptides of $N$. tabacum genes were added to precursor and mature Ag85B-CP to compare the efficiency of cytoplasmic and apoplastic expression. Constructs were agroinfiltrated into $N$. benthamiana leaves and the yield and integrity of recombinant proteins were analysed. Glycosylation status was determined by treatment with peptide N-glycosidase F. The highest amounts of fusion protein were obtained by expressing mature Ag85B lacking its leader sequence directed to the apoplast, which reached a yield of $100 \mathrm{mg}$ of antigen per $\mathrm{kg}$ of fresh leaf. Glycosylated and non-glycosylated fusion proteins were obtained in the apoplastic and cytoplasmic space, respectively. We showed the feasibility of producing Ag85B-CP protein in $N$. benthamiana leaves for application as a subunit vaccine and demonstrated the importance of expressing mature Ag85B to increase yield and to avoid the production of degraded protein fragments unsuitable for a pharmaceutical product.
\end{abstract}

Keywords: Ag85B, Agroinfiltration, Molecular Pharming, PVX-CP, Tuberculosis

\section{Introduction}

Tuberculosis (TB) is a re-emerging disease that represents one of the leading causes of death from a single infectious agent. It is a disease of poverty, affecting mostly young adults in their most productive years with the vast majority of TB cases occurring in developing countries. The current vaccine against the causal agent for human TB is a strain of the bovine pathogen Mycobacterium bovis, the Bacillus CalmetteGuerin (BCG). Even when there is consensus about its efficiency to protect against meningial TB in children, its efficiency to protect against lung infections in adults and its safety have been challenged (Moliva et al., 2015). Due to this, interest in the development of alternative TB vaccines has increased in past years, including protein subunit-based vaccines attempting to induce immunity by one or a combination of antigens.

Protein subunit-based vaccines are an interesting strategy for TB because, in contrast to BCG, they are not compromised by a previous exposure to mycobacteria and can also be retained as a booster to BCG priming to prolong immunity in adults (Andersen and Woodworth, 2014). Due to the intracellular nature of the infection by $M$. tuberculosis, it is important to find antigens capable of generating Th1-type stimulation in order to achieve protection. One of the strongest candidates to this end is antigen 85B (Ag85B), whose protective efficacy against $M$. tuberculosis has been extensive demonstrated. Efficient protection against bovine tuberculosis has also been shown in the context of DNA vaccines (Teixeira et al., 2006) and as a subunit vaccine in a murine and guinea pig model 
(Olsen et al., 2004; Aagard et al., 2009), making Ag85B a key antigen in the search of new vaccines directed against both human and bovine tuberculosis.

Plant-based expression systems are an attractive approach for the production and delivery of subunit-based vaccines (Bey et al., 2010; Komarova et al., 2010) and secretion-based systems can improve production by exploiting the natural secretion of some plant proteins to the extracellular root tissue (rhizosecretion) (Drake et al., 2009; Madeira et al., 2016). In addition, the production of antigens in the surface of plant Virus-Like Particles (VLPs) is a promising vaccine strategy, as they can improve safety and immunogenicity (Chen and Lai, 2013). The Coat Protein (CP) of potexviruses like the Potato Virus $X$ (PVX) is able to self-assemble into ordered VLPs that have no major size and packaging constraints, making them ideal carriers for harbouring antigens (Zelada et al., 2006; Bey et al., 2010; Plchova et al., 2011; Dickmeis et al., 2015).

Here, we explored different strategies to produce Ag85B for its potential use as a vaccine. We expressed Ag85B fused to PVX-CP in Nicotiana benthamiana leaves and found that guiding a mature version of the Ag85B protein to the apoplastic space improves yields and produces a glycosylated protein.

\section{Materials and Methods}

\section{Codon Optimization of Ag85B Sequence for Plant Expression}

The codons of the M. tuberculosis Ag85B sequence (GenBank ID AY207396.1) were optimized for efficient expression in $N$. benthamiana and $N$. tabacum. In silico optimization was achieved with OPTIMIZER software (Puigbò et al., 2007) using the one amino acid-one codon method. The codon usage data of $N$. tabacum and $N$. benthamiana were obtained from the CUTG (Codon Usage Tabulated from GenBank (http://www.kazusa.or.jp/codon/). Aditionally specific 6base restriction sites and $\mathrm{A}+\mathrm{T}$-rich destabilizing sequences were removed and the GC content was decreased from $63.6 \%$ in the original sequence to $39 \%$ in the optimized one. The plant-optimized Ag58B gene was chemically synthesized by GeneScript Inc (Piscataway, NJ, USA) and delivered as an insert in the pUC57 plasmid.

\section{Construction of Cytoplasmic and Apoplastic Targeting Vectors}

To construct the different expressing vectors (Fig. 2), the following sequences were obtained by chemical synthesis: (i) 5 ' untranslated region (UTR) of $N$. tabacum alcohol dehydrogenase containing a translational enhancer (GenBank ID AY619947; Satoh et al., 2004) followed by either the signal peptide of $N$. tabacum cationic peroxidase $38 \mathrm{~K}$ (38Psp, GenBank ID D42064) or the signal peptide of $N$. tabacum beta-D-glucan exohydrolase (GEsp, accession no. AB017502) flanked by recognition sites for Cla I; (ii) the plant-optimized Ag85B sequence flanked by Xho I sites and (iii) the sequence of the Potato virus $X$ coat protein (PVX-CP; GenBank ID P62406.1) flanked by $B g l$ II sites. The synthesized sequences were cloned into the pUC57 vector between the recombination sequences attL1 and attL2, generating donor vector pDONR-38Psp.Ag85B.CP for cloning using Gateway technology (Invitrogen). Vector pDONR-Ag85B.CP was obtained by Cla I digestion and religation of pDONR-38Psp.Ag85B.CP. To make vectors pDONR-Ag85Bm.CP and pDONR-38Psp.Ag85Bm.CP carrying Ag85B sequences that lack the leader region, inverse PCR was performed with primers 5'GGGGGGCCATGGCTTCAAGTTCCTTCTCCTTCTAT GG-3' and 5'GGGGGGCCATGGCATCTCGAGATCGATACCAGC3', using vectors pDONR-Ag85B.CP and pDONR-38PspAg85B.CP as templates. Vector pDONRGEsp.Ag85Bm.CP was obtained by replacing the 38Psp sequence in Cla I-digested pDONR-38Psp.Ag85Bm.CP with the chemically-synthesized GEsp sequence. Sequences were verified by automated sequencing. The pDONR vectors were recombined with the destiny binary vector pK7WG2D, which contains the duplicate cauliflower mosaic virus $35 \mathrm{~S}$ promoter ( $2 \mathrm{x} 35 \mathrm{SCaMV})$ and the nopaline synthase (nos) terminator to stop transcription.

The predicted molecular masses of fusion proteins were estimated using Expasy Bioinformatics website (https://web.expasy.org/compute_pi/). The signal peptide cleavage sites of secreted protein fusions were predicted using SignalP 4.1 software (Petersen et al., 2011). Sequences of the constructs are available from the authors upon request.

\section{Agrobacterium-Mediated Transient Expression Assays}

Each construct was transformed into A. tumefaciens GV3101 to perform agroinfiltration assays in $N$. benthamiana leaves. Agrobacterium cells were grown in LB-medium containing $100 \mathrm{mg} / \mathrm{L}$ rifampicin, $50 \mathrm{mg} / \mathrm{L}$ gentamicin and antibiotics suitable for each plasmid at $28^{\circ} \mathrm{C}$ until an $\mathrm{OD}=0.6$, collected by centrifugation and resuspended in water at $\mathrm{OD}=0.3$. Prior to infiltration, equal volumes of vector-containing Agrobacteria expressing Ag85B-CP fused proteins were mixed with those expressing the Tomato bushy stunt virus P19 (pBIN61-P19), a well known suppressor of posttranscriptional gene silencing (kindly provided by David Baulcombe, Sainsbury Laboratory, John Innes Centre Sainsbury, UK). Agroinjection was conducted on fully expanded leaves of the third and fourth node and bacterial suspension was injected using a $5 \mathrm{~mL}$ syringe. After injection, plants were further grown under greenhouse conditions and total proteins were extracted from infiltrated leaves after 4 days post infiltration and tested by Western blot using Ag85B and CP specific antibodies. 


\section{Western Blot}

Total protein was extracted from agroinfiltrated leaves, resolved by $12 \%$ SDS-PAGE and gels were blotted to nitrocellulose membrane using a semi-dry transfer system (Bio-Rad). Ponceau red staining of the large RuBisCO subunit was used as loading control. Membranes were blocked with $5 \% \mathrm{w} / \mathrm{v}$ non-fat milk in TBS containing $0.05 \% \mathrm{v} / \mathrm{v}$ Tween 20 (TBST-milk) during 2 hours. Detection was done with primary antibodies anti-Ag85B rabbit antiserum (1:500; kindly provided by Fabiana Bigi, INTA-Castelar, Argentina) and anti-PVX-CP (1:1,500; Adgen) and the second antibody anti-rabbit IgG goat antiserum coupled to horseradish peroxidase $(1: 10,000$; Sigma) diluted in TBST-milk. Blots were developed using a chemiluminescent system.

\section{Glycosylation Assays}

Crude protein extracts of 38Psp.Ag85B.CP and 38Psp.Ag85Bm.CP agroinfiltrated leaves were treated with Peptide N-Glycosidase F (PNGase F) following the manufacturer's instructions (New England BioLabs). Both treated and untreated extracts were analysed by SDS 12\% PAGE and Western blot against Ag85B.

\section{Statistical Analyses}

Prism 5.0 software (GraphPad Software Inc., San Diego, CA) was used for the data handling and results are presented as means \pm standard error of the mean (SEM). Between-group comparisons were performed by one-way ANOVA and Tukey's test and statistical significance was set at $\mathrm{p}<0.05$.

\section{Results}

\section{Construction of Binary Vectors for Ag85B Expression in Plants}

M. tuberculosis Ag85B is a secreted protein. It has a $\mathrm{N}$-terminal functional leader sequence which is cleaved off during export and release of the mature protein in mycobacterial culture filtrate (Malen et al., 2007). Taking this into account, we studied the expression of both precursor (Ag85B) and mature Ag85B (Ag85Bm) sequences in $N$. benthamiana. To maximize codon usage in a plant expression system, the DNA sequence encoding precursor Ag85B was subjected to codon optimization aided by the OPTIMIZER software (Puigbò et al., 2007), resulting in $78 \%$ of codons being altered while maintaining the amino acid sequence. The codon adaptation index (CAI) of optimized sequence increased from 0.552 to 1 and 0.407 to 0.92 for $N$. benthamiana and N. tabacum codon usage, respectively (Fig. 1).

Since it has been observed that antigens expressed in the context of viral-like particles can serve as better immunogens, we studied the feasibilty to express both $\mathrm{Ag} 58 \mathrm{~B}$ and $\mathrm{Ag} 85 \mathrm{Bm}$ fused to the coat protein of Potato
Virus $X$ (PVX-CP). In addition, we also decided to evaluate the expression level of chimeric antigens directed to the cytoplasmic or apoplastic compartments using a cassette validated for high secretory expression in N. tabacum plants (Matsui et al., 2006). Two different $N$. tabacum signal peptides were tested, namely one from the $38 \mathrm{kDa}$ cationic peroxidase isozyme (38Sp) and the other from the beta-D-glucan exohydrolase (GEsp). The constructs analysed in this study are shown in Fig. 2.

\section{Evaluation of Expression of Precursor and Mature Ag85B by Agroinfiltration}

Agrobacterium-mediated transient agroinfiltration systems was used to evaluate the expression of Ag85B constructs in $N$. benthamiana leaves. To enhance transient expression, each construct was co-infiltrated with $A$. tumefaciens harbouring a plasmid to express the Post-Transcriptional Gene Silencing (PTGS) suppressor TBSV-P19. Agroinfiltrated leaves were collected 4 days post infiltration (d.p.i.) and antigen expression was evaluated in total protein extracts by Western blot using anti-M. tuberculosis 85B and anti-PVX-CP antibodies.

Agroinfiltration with plasmid Ag85B.CP (Fig. 2), which should express the cytoplasmic version of the Ag85B-CP fusion protein, failed to produce significant levels of expressed protein, since anti-Ag85B and antiPVX-CP antibodies could not reveal any bands in Western blots (Fig. 3, lanes 2 and 8). On the other hand, the secreted version of the same fusion protein, expressed from plasmid 38Psp.Ag85B.CP (Fig. 2), was detected by both antibodies as two distinct bands, one major band of $30 \mathrm{kDa}$ and a minor band of around $70 \mathrm{kDa}$ (Fig. 3, lanes 3 and 9), none of which correspond to the band of around $60 \mathrm{kDa}$ expected for the Ag85B-CP fusion protein. Since the secreted version of the protein could undergo posttranslational modifications via the endomembrane system, the $70 \mathrm{kDa}$ band might correspond to a glycosylated version of the protein (see below), while the $30 \mathrm{kDa}$ band could represent a proteolytic fragment. Other minor bands detected by both antibodies might also correspond to proteolytic fragments.

The influence of the mycobacterial leader sequence in plant expression was evaluated with constructs carrying the mature sequence of $\mathrm{Ag} 85 \mathrm{~B}$ (Ag85Bm), i.e., lacking the leader sequence. The construct expressing the cytoplasmic version of the Ag85Bm-CP fusion protein (Ag85Bm.CP; Fig. 2) yielded a specific protein of around $55 \mathrm{kDa}$ recognized by anti-Ag85B and antiPVX-CP antibodies, which corresponds to the expected size of intact cytoplasmic Ag85Bm-CP (Fig. 3, lanes 4 and 10). Thus, the presence of the native mycobacterial leader sequence seems to be deleterious to cytoplasmic Ag85B-CP expression (compare lanes 2 and 4 and lanes 8 and 10 in Fig. 3). When Ag85Bm-CP was directed to the apoplast using two different $N$. tabacum signal peptides (38Psp.Ag85Bm.CP and GEsp.Ag85Bm.CP; Fig. 2), a protein of around $70 \mathrm{kDa}$ was detected by 
Western blot (Fig. 3; lanes 5, 6, 11 and 12). In addition, the protein yields of secreted proteins were much higher than those of the cytoplasmic version (Fig. 3, compare lane 4 with lanes 5 and 6 and lane 10 with lanes 11 and 12). Importantly, the secreted Ag85Bm-CP protein was mostly intact, without the major proteolytic fragment that was detected when the leader sequence was left in the Ag85B protein (Fig. 3, compare lane 3 with lanes 5 and 6 and lane 9 with lanes 11 and 12). Specifically, densitometric analyses indicate that, compared to the 55
kDa cytoplasmic version, yields could be increased 8 fold and 5 fold when Ag85Bm-CP was expressed using the 38Psp and the GEsp signal peptides, respectively (Fig. 4B). When guided to the apoplast, the yield of mature Ag85B expressed with the 38Psp signal peptide was 2 -fold higher than that of precursor Ag85B (Fig. 4B). Thus, directing a Ag85B fusion protein lacking the mycobacterial leader sequence to the apoplastic space is the optimal condition for the expression of Ag85B in $N$. benthamiana leaves by agroinfiltration.
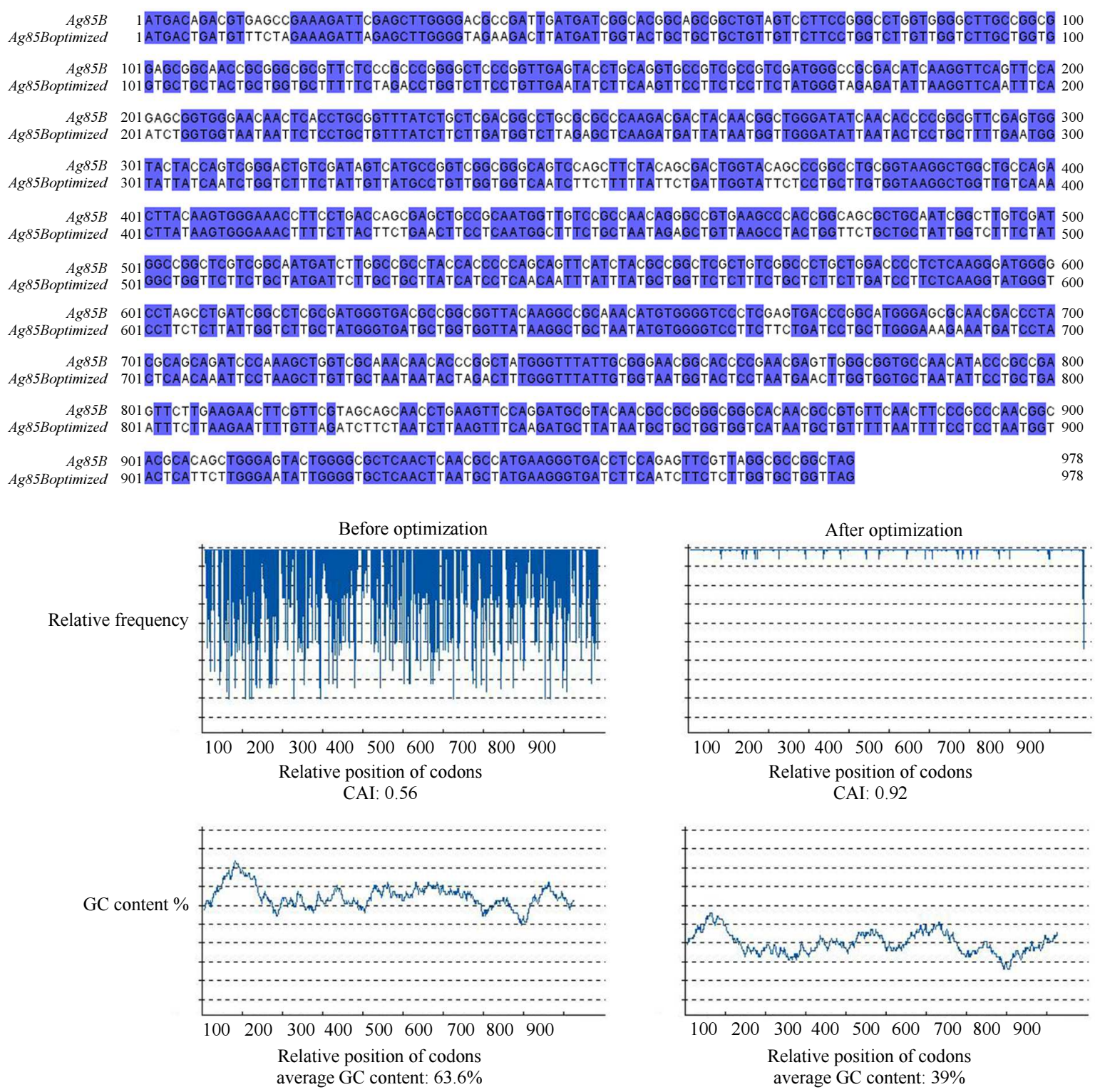

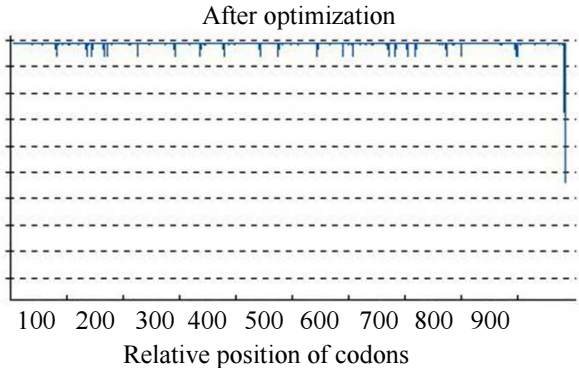

CAI: 0.92

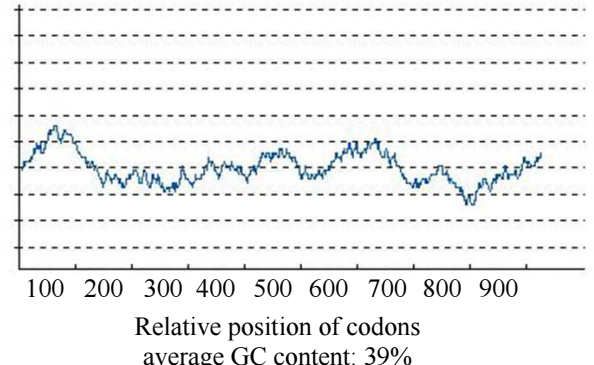

Fig. 1: Codon optimization of M. tuberculosis Ag85B sequence for expression in plants. Upper panel shows an alignment of Ag85B (AY207396.1) and optimized Ag85B sequences, with identical nucleotides highlighted in blue and modified nucleotides in white background. Middle panel shows the distribution of codon usage frequency along the length of the Ag85B gene sequence before (left) and after (right) optimization for expression in N. tabacum. The Codon Adaptation Index (CAI) was increased from 0.56 to 0.92 (the frequency of the non-optimized bases is decreased after optimization). Lower panels show that the overall GC content was reduced from 63.6 to $39 \%$ in the optimized sequence 


\section{Ag85B.CP}

\begin{tabular}{|lll|}
\hline 5'UTR $\quad$ LS & Ag85B & PVX-CP \\
\hline & & \\
\hline
\end{tabular}

$60 \mathrm{kDa}$

38Psp.Ag85B.CP

\begin{tabular}{ll} 
5'UTR & 38 Psp LS \\
\hline & \&g: \\
\hline
\end{tabular}

Ag85B
PVX-CP

1

$60.5 \mathrm{kDa}$

Ag85Bm.CP

\begin{tabular}{|l|l|l|}
\multicolumn{1}{l|}{ 5'UTR } & Ag85Bm & \multicolumn{1}{c}{ PVX-CP } \\
\hline & & \\
\hline
\end{tabular}

$55 \mathrm{kDa}$

38Psp.Ag85Bm.CP

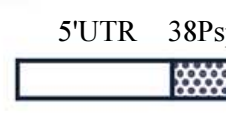

PVX-CP

$\mathrm{PVX}-\mathrm{CP}$

$55.6 \mathrm{kDa}$

\begin{tabular}{lrrr|r} 
& 5'UTR & GEsp & Ag85Bm & \multicolumn{2}{c}{ PVX-CP } \\
\cline { 2 - 4 } GEsp.Ag85Bm.CP & & & \\
\cline { 2 - 4 } & & & &
\end{tabular}

Fig. 2: Schematic representation of constructs used in this study. All expression cassettes are cloned into the pK7WG2D binary vector. Gene expression is under the control of the CaMV 35S promoter, the $N$. tabacum alcohol deshydrogenase 5'-UTR and the nopaline synthase terminator sequence. Ag85B: precursor Ag85B sequence; Ag85Bm: mature Ag85B sequence; LS: bacterial leader sequence of Ag85B precursor; 38Psp: $N$. tabacum 38 kDa cationic peroxidase peptide signal; GEsp: N. tabacum beta-D-glucan exohydrolase peptide signal; PVX-CP: Potato virus $X$ coat protein sequence. The predicted molecular masses of fusion proteins are shown to the right. For secreted fusion proteins, the molecular masses of the mature proteins are indicated

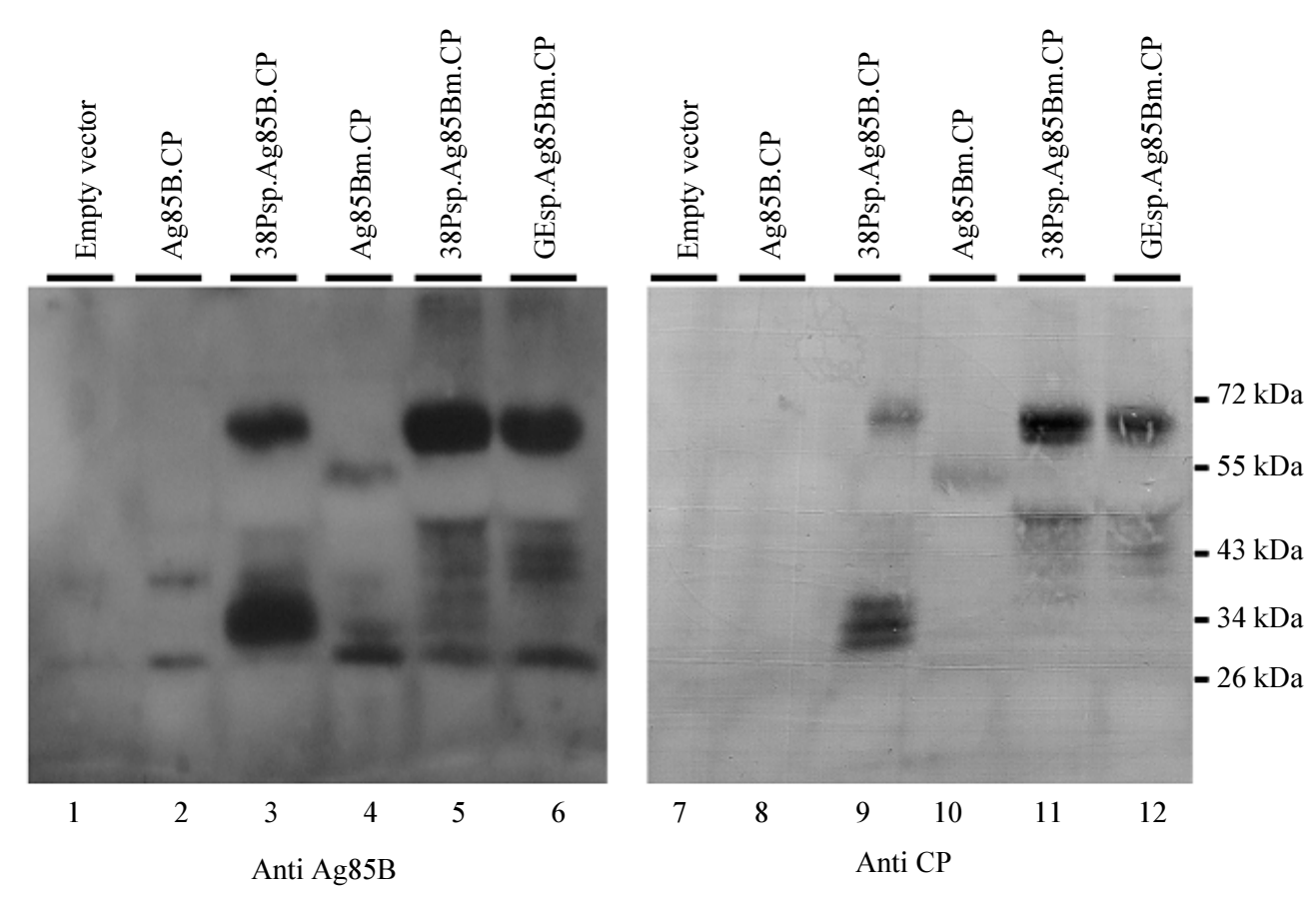

Fig. 3: Expression of the Ag85B-CP in $N$. benthamiana plants by agroinfiltration. Total proteins were extracted from leaves agroinfiltrated with different constructions after $4 \mathrm{dpi}$, resolved in SDS PAGE and detected by Western blot. Left panel: Immunoblot with anti-Ag85B rabbit antiserum. Right panel: Immunoblot with an anti-PVX-CP rabbit antiserum. Proteins from leaves co-agroinfiltrated with empty vector and P19 were used as negative control. Positions of pre-stained protein weight markers are indicated at the right side of the panels 


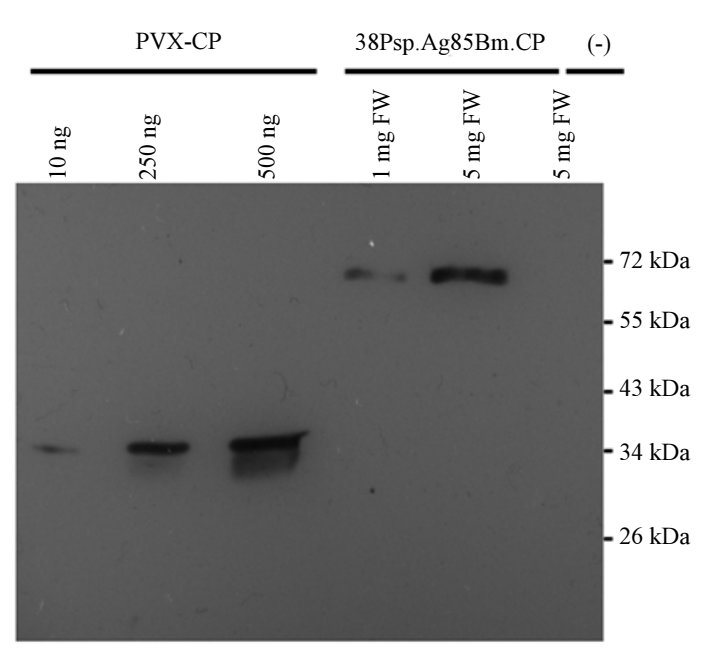

(A)

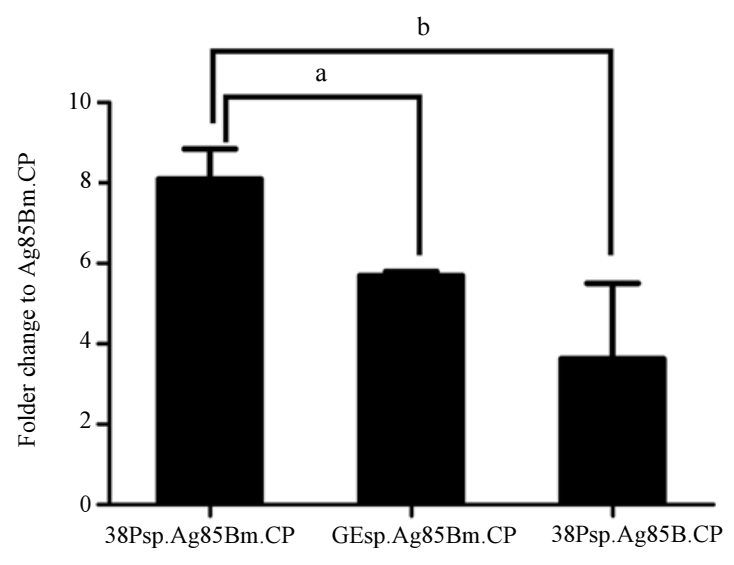

(B)

Fig. 4: Quantification of $\mathrm{Ag} 85 \mathrm{Bm}-\mathrm{CP}$ and $\mathrm{Ag} 85 \mathrm{~B}-\mathrm{CP}$ produced by transient expression in N. benthamiana plants. (A) Leaf extracts containing $1 \mathrm{mg}$ and $5 \mathrm{mg}$ of total soluble protein (TSP) from plants agroinfiltrated with 38Psp.Ag85Bm.CP vector were subjected to Western blot analysis using anti-PVX-CP rabbit antiserum and a anti-rabbit IgG goat antiserum coupled to horseradish peroxidase. Different amounts of purified, bacterially expressed PVX-CP were included as mass reference. Proteins from leaves co-agroinfiltrated with empty vector and P19 were used as negative control (-). Positions of pre-stained protein weight markers are indicated at the right side of the panel. (B) Graph shows the fold-increase in the expression levels of apoplastic-expressed Ag85Bm-CP (38Psp.Ag85Bm.CP and GEsp.Ag85Bm.CP) and Ag85B-CP (38Psp.Ag85B.CP) in relation to cytoplasmic-expressed Ag85Bm-CP. Band densitometry was performed using the Image $\mathrm{J}$ software. Bars correspond to standard error of the mean (SEM) of three experiments. Statistically significant difference between groups was determined by one-way ANOVA followed by Turkey's test. Pair of groups that display statistically significant differences are indicated $(\mathrm{a}, \mathrm{p}=0.0449 ; \mathrm{b}, \mathrm{p}=0.0026)$

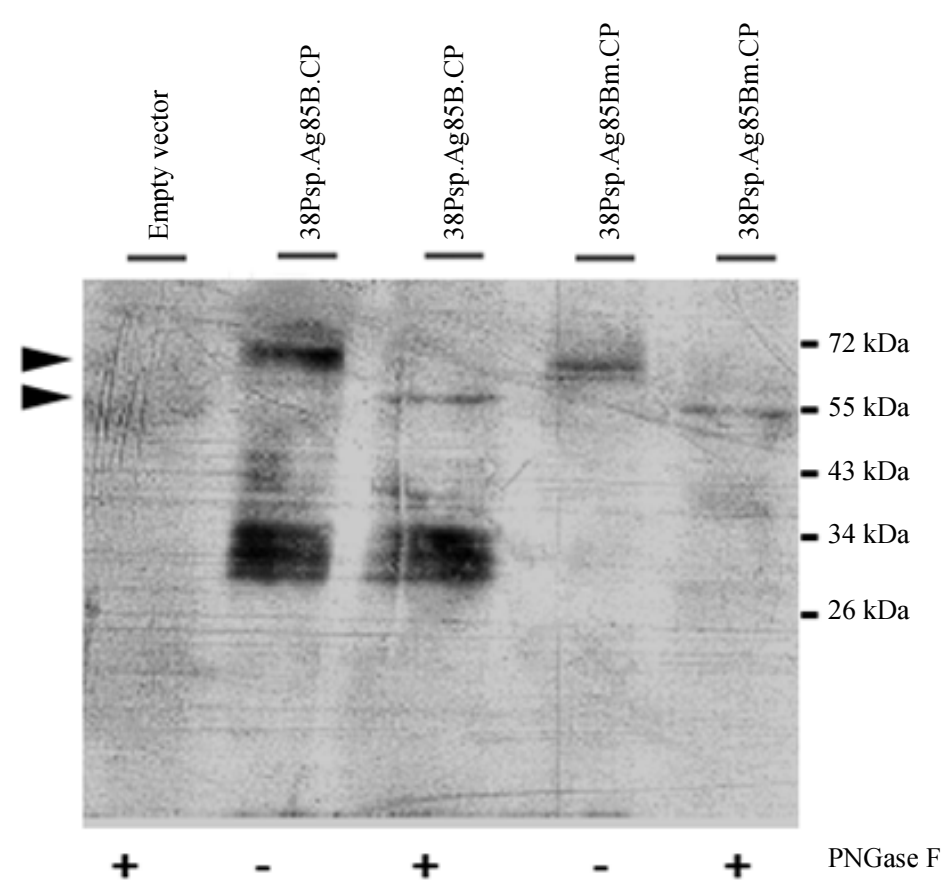

Fig. 5: N-glycosylation of the Ag85Bm-CP protein. Leaf extracts containing $1 \mathrm{mg}$ of total soluble protein (TSP) from plants agroinfiltrated with 38Psp.Ag85B-CP or 38Psp.Ag85Bm-CP were treated (+) or non-treated (-) with PNGase F and subjected to Western blot with an anti-Ag85B rabbit antiserum and an anti-rabbit IgG goat antiserum coupled to horseradish peroxidase. Position of pre-stained protein weight markers is indicated at the right side of the panel 
To estimate the level of antigen accumulation produced from the highest yielding construction, namely 38Psp.Ag85Bm.CP, a Western blot was carried out with an anti-PVX-CP antiserum and the density of the appropriate band was compared with a standard curve of known amounts of bacterially-expressed PVX-CP (Fig. 4A). The levels of Ag85Bm-CP antigen were calculated to be $100 \mathrm{mg} / \mathrm{kg}$ of fresh leaf weight using the Image J software (Schneider et al., 2012).

\section{Glycosylation Analysis of Apoplastic Ag85B Fusion Proteins}

As mentioned earlier, the secreted versions of Ag85B-CP and Ag85Bm-CP displayed apparent molecular masses of around $70 \mathrm{kDa}$, which are higher than the predicted $60 \mathrm{kDa}$ and $55 \mathrm{kDa}$, respectively. It has been previously shown that Ag85B is Nglycosylated in mammalian cells when targeted to the secretory pathway via the endomembrane system (Malin et al., 2000). In order to check whether the observed shift in electrophoretic mobility in the secreted proteins could be due to $\mathrm{N}$-glycosylation, we treated crude protein extracts of 38Psp.Ag85B.CP and 38Psp.Ag85Bm.CP-agroinfiltrated leaves with peptide N-glycosidase F (PNGase F). Both treated and untreated extracts were analysed by SDS 12\% PAGE and Western blot against Ag85B. After treatment, Ag85B-CP and Ag85Bm-CP diminished their electrophoretic mobilities, becoming similar to that of the respective cytoplasmic version of the protein (Fig. 5, compare lanes 2 and 3 and 4 and 5). This confirms that the secreted versions of Ag85B fusion protein are $\mathrm{N}$-glycosylated, regardless of the presence of the leader sequence.

\section{Discussion}

Transient gene expression in plants by agroinfiltration is a powerful plataform to produce antigens due to its low-cost, putative high protein yield and scalability. It is also a rapid method to evaluate the adequacy of expressing a particular antigen in a plant system before the time-consuming generation of transgenic plants. Using this approach, we tested the feasibility of expressing Ag85B fused to PVX-CP for use as an immunogen against tuberculosis. $\mathrm{Ag} 85 \mathrm{~B}$ is efficiently secreted in mycobacterial culture filtrate as a 30-kDa mature protein (Malen et al., 2007), which led us to evaluate the impact of the leader sequence on antigen production and integrity. Previously, it was shown that the deletion of a hydrophobic sequence contained within the leader sequence, dubbed "transmembrane domain", increased the production of cytoplasmic Ag85B fusion protein, but the production of a secreted version of the protein was not attempted (Dorokhov et al., 2007). Another study showed that Ag85B fusion protein could be expressed when directed to the endoplasmic reticulum, but Ag85B was expressed with its leader sequence and the recombinant antigen exhibited evidence of protein degradation (Floss et al., 2010). Here, we show for the first time the importance of expressing the mature sequence of $\mathrm{Ag} 85 \mathrm{~B}$ to obtain homogeneous and intact antigen in plants both in the cytoplasm and in the apoplastic space. Importantly, it is the mature sequence, not the leader sequence, that contains the main immunodominant T-cell epitopes of Ag85B relevant in eliciting an immune response (D'Souza et al., 2003; Huygen, 2014).

We demonstrated that Ag85B and Ag85Bm secreted fusion protein undergoes $\mathrm{N}$-glycosylation, an interesting result since glycosylation in plants can potentially enhance the way an antigen is taken up, degraded and presented to cells of the adaptive immune system (Bosch and Schots, 2010). In line with this, it has been shown that the expression of $\mathrm{Ag} 85 \mathrm{~B}$ with tissue plasminogen activator signal peptide in mammalian cell lines results in a non-natural $\mathrm{N}$-glycosylated version of $\mathrm{Ag} 85 \mathrm{~B}$ that exhibits increased immunogenicity (Malin et al., 2000). Interestingly, an analysis of potential N-glycosylated sites in Agb85B using the GlycoEP software (Chauhan et al., 2013) indicates the presence of two high-scoring potential Nglycosylation sites (N243 and N253), both of which are located within the main T-cell immunodominant epitopes of Ag85B (peptide 240-260) (Huygen, 2014). The capacity to produce glycosylated and nonglycosylated mature $\mathrm{Ag} 85 \mathrm{~B}$ fused to $\mathrm{CP}$ in plants opens the possibility for further studies on the impact of $\mathrm{N}$ glycosylation on Ag85B immunogenicity.

\section{Conclusion}

In conclusion, we showed that transient expression with concomitant suppression of post-transcriptional gene silencing represents a technology for fast and inexpensive production of $\mathrm{Ag} 85 \mathrm{~B}$ fused to $\mathrm{CP}$ antigens using $N$. benthamiana plants. We were able to enhance the accumulation levels of Ag85B fused to $\mathrm{CP}$ by targeting the fusion protein to the apoplast compartment and deleting the N-terminal native leader sequence. The screening allowed us to choose the best candidate for the production of $\mathrm{Ag} 85 \mathrm{~B}$ in transgenic plants by rhizosecretion and study the formation of VLPs harbouring Ag85B at the surface.

\section{Acknowledgement}

We thank Dr. David Baulcombe (Sainsbury Laboratory, Norfolk, UK) for the P19 suppressor and Dr. Fabiana Bigi (Instituto Nacional de Tecnología Agropecuaria, Castelar, Argentina) for the antiAg85B antibody. 


\section{Funding Information}

This work was supported by grants from the National Agency for the Promotion of Science and Technology of Argentina (ANPCyT, PICT 05-32232). A.Z. is a career research scientist from CONICET. P.A.G. and F.P. are ANPCyT and CONICET fellows.

\section{Author's Contributions}

Pablo A Gonzalez: Conceived and performed the experiments.

Franco D Puccio: Performed some of the experiments.

Alicia M Zelada: Conceived and designed the experiments, analysed the data and wrote the manuscript.

\section{Ethics}

The authors declare no conflicts of interest.

\section{References}

Aagard, C., T.T. Hoang, A. Izzo, R. Billeskov and J. Troudt et al., 2009. Protection and polyfunctional $\mathrm{T}$ cells induced by Ag85B-TB10.4/IC31 against Mycobacterium tuberculosis is highly dependent on the antigen dose. PLoS One, 4: e5930-e5930.

DOI: 10.1371/journal.pone.0005930

Andersen, P. and J.S. Woodworth, 2014. Tuberculosis vaccines--rethinking the current paradigm. Trends Immunol., 35: 387-395.

DOI: $10.1016 /$ j.it.2014.04.006

Bey, P., M. Binaghi, A.N. Mentaberry and A.M. Zelada, 2010. Plant viral vectors as a tool for vaccine production. Am. J. Plant Sci. Biotechnol., 4: 90-97.

Bosch, D. and A. Schots, 2010. Plant glycans: Friend or foe in vaccine development? Expert Rev. Vaccines, 9: 835-842. DOI: $10.1586 /$ erv. 10.83

Chauhan, J.S., A. Rao and G.P.S. Raghava, 2013. In silico platform for prediction of $\mathrm{N}-, \mathrm{O}-$ and $\mathrm{C}$ glycosites in eukaryotic protein sequences. PLoS One, 8: e67008-e67008.

DOI: 10.1371/journal.pone.0067008

Chen, Q. and H. Lai, 2013. Plant-derived virus-like particles as vaccines. Hum. Vaccin. Immunother., 9: 26-49. DOI: $10.4161 / \mathrm{hv} .22218$

Dickmeis, C., M.M. Honickel, R. Fischer and U. Commandeur, 2015. Production of hybrid chimeric PVX particles using a combination of TMV and PVXbased expression vectors. Front Bioeng. Biotechnol., 3: 189-189. DOI: 10.3389/fbioe.2015.00189

Dorokhov, Y.L., A.A. Sheveleva, O.Y. Frolova, T.V. Komarova and A.S. Zvereva et al., 2007. Superexpression of tuberculosis antigens in plant leaves. Tuberculosis, 87: 218-224.

DOI: $10.1016 /$ j.tube.2006.10.001
Drake, P.M., T. Barbi, A. Sexton, E. McGowan and J. Stadlmann et al., 2009. Development of rhizosecretion as a production system for recombinant proteins from hydroponic cultivated tobacco. FASEB J., 23: 3581-3589. DOI: 10.1096/fj.09-131771

D'Souza, S., V. Rosseels, M. Romano, A. Tanghe and O. Denis et al., 2003. Mapping of murine Th1 Helper T-cell epitopes of mycolyl transferases Ag85A, Ag85B and Ag85C from Mycobacterium tuberculosis. Infect. Immun., 71: 483-493.

DOI: 10.1128/IAI.71.1.483-493.2003

Floss, D.M., M. Mockey, G. Zanello, D. Brosson and M. Diogon et al., 2010. Expression and immunogenicity of the mycobacterial Ag85B/ESAT-6 antigens produced in transgenic plants by elastin-like peptide fusion strategy. J. Biomed. Biotechnol., 27: 43-46. DOI: $10.1155 / 2010 / 274346$

Huygen, K., 2014. The immunodominant T-cell epitopes of the mycolyl-transferases of the antigen 85 complex of M. tuberculosis. Front Immunol., 5: 321. DOI: 10.3389/fimmu.2014.00321

Komarova, T.V., S. Baschieri, M. Donini, C. Marusic and E. Benvenuto et al., 2010. Transient expression systems for plant-derived biopharmaceuticals. Expert Rev. Vaccines, 9: 859-876. DOI: $10.1586 /$ erv. 10.85

Madeira, L.M., T.H. Szeto, M. Henquet, N. Raven and J. Runions et al., 2016. High-yield production of a human monoclonal $\mathrm{IgG}$ by rhizosecretion in hydroponic tobacco cultures. Plant Biotechnol. J., 14: 615-24. DOI: 10.1111/pbi.12407

Malen, H., F.S. Berven, K.E. Fladmark and H.G. Wiker, 2007. Comprehensive analysis of exported proteins from Mycobacterium tuberculosis H37Rv. Proteomics, 7: 1702-1718. DOI: $10.1002 /$ pmic. 200600853

Malin, A.S., K. Huygen, J. Content, M. Mackett and L. Brandt et al., 2000. Vaccinia expression of Mycobacterium tuberculosis-secreted proteins: Tissue plasminogen activator signal sequence enhances expression and immunogenicity of $\mathrm{M}$. tuberculosis Ag85. Microbes Infect., 2: 1677-1685. DOI: $10.1016 / \mathrm{S} 1286-4579(00) 01323-\mathrm{X}$

Matsui, T., M. Hori, N. Shizawa, H. Nakayama and A. Shinmyo et al., 2006. High-efficiency secretory production of peroxidase $\mathrm{Cla}$ using vesicular transport engineering in transgenic tobacco. J. Biosci. Bioeng., 102: 102-109. DOI: 10.1263/jbb.102.102

Moliva, J.I., J. Turner and J.B. Torrelles, 2015. Prospects in Mycobacterium bovis Bacille Calmette et Guérin (BCG) vaccine diversity and delivery: Why does $\mathrm{BCG}$ fail to protect against tuberculosis? Vaccine, 33: 5035-5041. DOI: $10.1016 /$ j.vaccine.2015.08.033 
Olsen, A.W., A. Williams, L.M. Okkels, G. Hatch and P. Andersen, 2004. Protective effect of a tuberculosis subunit vaccine based on a fusion of antigen $85 \mathrm{~B}$ and ESAT-6 in the aerosol guinea pig model. Infect. Immun., 72: 6148-6150. DOI: $10.1128 /$ IAI.72.10.6148-6150.2004

Petersen, T.N., S. Brunak, G. von Heijne and H. Nielsen, 2011. SignalP 4.0: Discriminating signal peptides from transmembrane regions. Nat. Meth., 8: 785-786. DOI: $10.1038 /$ nmeth. 1701

Puigbò, P., E. Guzmán, A. Romeu and S. GarciaVallvé, 2007. OPTIMIZER: A web server for optimizing the codon usage of DNA sequences. Nucl. Acid Res., 35: W126-W131.

DOI: $10.1093 /$ nar/gkm219

Plchova, H., T. Moravec, H. Hoffmeisterova, J. Folwarczna and N. Cerovska, 2011. Expression of Human papillomavirus 16 E7ggg oncoprotein on $\mathrm{N}$ - and C-terminus of Potato virus $X$ coat protein in bacterial and plant cells. Protein Expr. Purif., 77: 146-152. DOI: 10.1016/j.pep.2011.01.008
Satoh, J., K. Kato and A. Shinmyo, 2004. The 5untranslated region of the tobacco alchol dehydrogenase gene functions as an effective translational enhancer in plant. J. Biosci. Bioeng., 98: 1-8. DOI: $10.1263 / \mathrm{jbb} .102 .102$

Schneider, C.A., W.S. Rasband and K.W. Eliceiri, 2012. NIH Image to ImageJ: 25 years of image analysis. Nat Methods. 9: 671-675.

Teixeira, F.M., H.C. Teixeira, A.P. Ferreira, M.F. Rodrigues and V. Azevedo et al., 2006. DNA vaccine using Mycobacterium bovis Ag85B antigen induces partial protection against experimental infection in BALB/c mice. Clin. Vaccine Immunol., 13: 930-935. DOI: 10.1128/CVI.00151-06

Zelada, A.M., G. Calamante, M. de la Paz Santangelo, F. Bigi and F. Verna et al., 2006. Expression of tuberculosis antigen ESAT-6 in Nicotiana tabacum using a potato virus X-based vector. Tuberculosis (Edinb), 86: 263-267.

DOI: $10.1016 /$ j.tube.2006.01.003 